\title{
Mixed Emotions, but Not Positive or Negative Emotions, Facilitate Legitimate Virus-Prevention Behaviors and Eudaimonic Outcomes in the Emergence of the COVID-19 Crisis
}

\author{
Vincent Y. S. $\mathrm{Oh}^{1}$ (D) $\cdot$ Eddie M. W. Tong ${ }^{1}$ \\ Received: 6 October 2020 / Accepted: 11 March 2021 / Published online: 20 April 2021 \\ (C) The Society for Affective Science 2021
}

\begin{abstract}
We report four studies ( $N=1419)$ examining emotional reactions from March to April 2020, when COVID-19 exhibited exponentially increasing infections and fatalities. Specifically, we examined associations between emotions with self-reported intentions to enact virus-prevention behaviors that protect oneself from COVID-19 and eudaimonic functioning. Study 1A, 1B, and Study 2 provided naturalistic evidence that mixed emotions predicted legitimate virus-prevention behaviors and eudaimonic functioning in the USA and Singapore, and Study 2 also supported receptivity as a mediator. Finally, Study 3 provided experimental evidence that mixed emotions causally increased legitimate virus-prevention behaviors relative to neutral, positive emotion, and negative emotion conditions, whereas eudaimonic functioning was increased only relative to the neutral condition. Across all studies, positive and negative emotions were unrelated to legitimate virus-prevention behaviors, while relationships with eudaimonic functioning were inconsistent. While self-reported measures do not represent actual behaviors, the findings suggest a potential role for mixed emotions in pandemic-related outcomes.
\end{abstract}

Keywords COVID-19 $\cdot$ mixed emotions $\cdot$ virus-prevention behaviors $\cdot$ eudaimonic well-being

The COVID-19 outbreak is an unprecedented global crisis which has exceeded millions of infections and fatalities worldwide. Amidst this crisis, two practical concerns are pertinent. The first is encouraging intentions to enact virus-prevention practices that could slow the spread of COVID-19, such as good hygiene and social distancing (Dalton et al., 2020). Complicating this, misinformation is widespread (Garrett, 2020) and has been propagated even by some authorities. The second concern is whether individuals can experience positive psychological outcomes despite the pandemic. It is hence pertinent to examine intentions to enact legitimate virus-prevention behaviors that may effectively prevent infection, as well as whether individuals can maintain positive functioning amidst this crisis. We examined these issues with

Handling editor: Nnamdi Pole

Vincent Y. S. Oh

vincent.ohys@u.nus.edu

1 Department of Psychology, National University of Singapore, Singapore, Singapore four studies from March to April 2020, when trajectories of infections and fatalities were rising exponentially.

In particular, the COVID-19 pandemic has been characterized by intense emotionality. As emotions are major antecedents of perceptions and behaviors (Keltner \& Gross, 1999), examining how emotions during this crisis may influence behaviors is crucial. Furthermore, mixed emotions, in which people simultaneously experience positive and negative emotions (Larsen \& McGraw, 2011), may dominate the emotional landscape of many individuals during this crisis (McCarthy, 2020). Whereas positive emotions may promote resilience (Gloria \& Steinhardt, 2016) and negative emotions generally have debilitative consequences (Badour et al., 2017), the role of mixed emotions in an international crisis like the COVID19 pandemic remains unknown and merits study.

One perspective, which we term the integrative perspective, argues that mixed emotions serve adaptive integrative functions, especially in adversity (Adler \& Hershfield, 2012; Larsen et al., 2003). For example, Rees et al. (2013) found across four experimental studies that mixed emotions facilitated better judgement on estimation tasks, and this was mediated by receptivity, which reflects a willingness to accept information from multiple perspectives even if they are 
conflicting. By facilitating receptivity and effective integration of divergent information, mixed emotions may promote complex thought processes (Fong, 2006) and well-being (Berrios et al., 2018). Moreover, these effects were unique from positive and negative emotions, suggesting that mixed emotions are distinctive affective states (Vaccaro et al., 2020). Although positive emotions may also broaden thought processes (Fredrickson, 2001), they may encourage uncritical acceptance of pro-attitudinal or mood-congruent information (Forgas \& East, 2008; Ziegler, 2014). Furthermore, while negative emotions may reduce gullibility (Forgas, 2019), they also encourage impulsivity (Selby et al., 2016) and ineffective coping (Tran et al., 2019). Mixed emotions may hence have unique implications from positive and negative emotions.

However, an alternative view of mixed emotions, which we term the conflict perspective, suggests that mixed emotions are conflicting and agonizing states (Mejía \& Hooker, 2017; van Harreveld et al., 2009) that are linked to negative traits, negative life events, and negative emotionality (Barford et al., 2020; Barford \& Smillie, 2016; Hui et al., 2009). Indeed, some evidence suggests that mixed emotions could be linked to motivational and behavioral avoidance (Durso et al., 2016; van Harreveld et al., 2015) and could have negative implications for self-esteem and well-being (Newman et al., 2019). These diverging theoretical perspectives complicate predictions concerning whether mixed emotions would be adaptive within the pandemic.

For example, the integrative perspective would predict that mixed emotions should facilitate receptivity towards legitimate virus-prevention behaviors, which are scientifically supported behaviors that may protect oneself from infection (e.g., social distancing). Conversely, the conflict perspective would predict that mixed emotions could encourage behavioral avoidance and reduce receptivity towards such behaviors. It is also unclear whether effects predicted by either perspective could generalize to unsupported virus-prevention behaviors, which may appear to have protective functions but are not scientifically supported (e.g., consuming honey), and atypical virus-prevention behaviors, which are not scientifically supported and are unusual behaviors that could be harmful (e.g., using detergent as mouthwash). While the finding that mixed emotions enable better judgement (Rees et al., 2013) implies that mixed emotions should not elicit indiscriminate receptivity to misinformation, the finding that mixed emotions consume regulatory resources (van Harreveld et al., 2009) could imply that mixed emotions may decrease the ability to inhibit maladaptive forms of coping.

Moreover, whereas the integrative perspective would predict that mixed emotions may enhance psychological functioning, the conflict perspective would predict that mixed emotions could interfere with psychological functioning. Specifically, we focused on eudaimonic functioning, which broadly encompasses positive functioning and flourishing.
Whereas hedonic conceptualizations focus on pleasure or the absence of adversity, eudaimonic conceptualizations focus on positive functioning even during adversity (Ryan \& Deci, 2001) and are especially appropriate for studying pandemicrelated outcomes. Three aspects of eudaimonia may be particularly relevant to this crisis: feeling appreciation towards life (Huta, 2013), developing complex insights about life (Bauer \& McAdams, 2010), and experiencing self-improvement motivations (Huta \& Waterman, 2014).

Given the conflicting theoretical perspectives, we made no a priori hypotheses and instead sought to provide empirical tests to determine which of the two competing perspectives would be supported by the data before examining the replicability of the findings. We conducted four studies from March to April 2020, focusing primarily on the USA, where the pandemic was in its early stages of exponentially worsening. Moreover, as argued by Dejonckheere et al., (2019), positive and negative emotions must be controlled for to conclude that any findings are independently and uniquely explained by mixed emotions. We hence examined global positive, negative, and mixed affective states to comprehensively examine emotional states and important pandemic-related outcomes, specifically legitimate, unsupported, and atypical virusprevention behaviors, and eudaimonic functioning. Furthermore, we adjusted for demographical variables that could be implicated in pandemic-related outcomes (e.g., Pasion et al., 2020), including age, gender, education level, household income, and household size, as well as social desirability, which may lead to artificial responses motivated by self-presentational concerns (Timmons et al., 2020). These controls ensure that findings for mixed emotions are not confounded by single-valenced emotions and demographical variability.

\section{Study 1}

Study 1 examined whether naturalistic emotions during the COVID-19 pandemic would predict virus-prevention behaviors and eudaimonic functioning. Specifically, Study $1 \mathrm{~A}$ was conducted in the USA between 26 March (85,356 infections) and 29 March (140,904 infections). Study 1B was conducted in Singapore, an East Asian country, between 26 March (683 infections) and 20 April (8014 infections), ${ }^{1}$ and serves as a comparison to determine whether the findings would generalize across two samples differing in culture and the pandemic's severity, among other differences.

\footnotetext{
${ }^{1}$ Statistics on cases and fatalities are retrieved from https://www.cdc.gov/ coronavirus/2019-ncov/cases-updates/previouscases.html and https:// covidsitrep.moh.gov.sg/.
} 


\section{Method}

\section{Participants}

We aimed to collect at least 300 participants to provide adequately powered tests for structural equation modelling (Kline, 2016). We recruited 351 participants from the USA with at least a 99\% approval rate and at least 10,000 HITs completed via Amazon Mechanical Turk (MTurk) for Study 1A, while 466 participants from Singapore were recruited via online advertisements for Study 1B. Participants in Study 1A were reimbursed with USD \$1.00; participants from Study 1B participated for a lucky draw of two SGD $\$ 100$ prizes. At the end of the study, participants were given a thorough debriefing (see Appendix C) to correct misinformation and direct them to reliable sources of information about the pandemic. We excluded 13 and 53 participants in Study 1A and Study 1B, respectively, for failing attention checks, giving 338 participants and 413 participants, respectively (see Table 1 for demographic details and demographic differences). Data and $R$ codes are available at https://osf.io/ tbwc5/?view_only=2cc3ae50f0f24f91ba7d206b21381d15.

\section{Measures}

Cronbach's alphas for all measures are presented in Table 1. Materials and scale construction considerations (including items, instructions, and psychometric properties) are provided in Appendix A.

Emotions Participants were instructed to think about the ongoing COVID-19 virus outbreak and asked "Over the past two weeks, to what extent have you felt the following emotions as a result of this outbreak?". The reference period of 2 weeks was specified to capture greater variability in emotions but also to avoid tapping into schematic or dispositional beliefs (e.g., Robinson \& Clore, 2002), and the outbreak was specified as the target to avoid assessing diffuse mood states rather than emotions (Beedie et al., 2005). They rated several emotion items presented in randomized order on a seven-point scale, with the following anchors: 1 ("Did not feel the emotion at all"), 4 ("Felt the emotion moderately"), and 7 ("Felt the emotion very much"). Positive emotions were measured by 21 items (e.g., "Grateful," "Joyful"); negative emotions were measured by 20 items (e.g., "Sad," "Fearful"). Mixed emotions were measured by 28 items adapted based on Barford and Smillie (2016; e.g., "a mixture of positive and negative emotions at the same time") and were rated on the same 7-point scale with the same anchors.

Virus-Prevention Behaviors We measured legitimate, unsupported, and atypical virus-prevention behaviors. Participants were given a list of behaviors and were told that these were coping behaviors that may have potential in reducing the risk of COVID-19. They then rated how likely they were to try each behavior to prevent getting infected by COVID-19 on a seven-point scale, with these anchors: 1 ("Not at all likely to do this"), 4 ("Somewhat likely to do this"), and 7 ("Very likely to do this"). Six items measured legitimate virus-prevention behaviors (e.g., "Wash your hands with soap or hand sanitizer frequently," "Minimize unnecessary social contact, such as social gatherings or sharing food with others"). Six items measured unsupported virus-prevention behaviors (e.g., "Exercise more frequently," "Consume honey regularly"). Six items measured atypical virus-prevention behaviors (e.g., "Rinse your mouth with detergent," "Take pills for malaria which may have antiviral properties").

Eudaimonic Functioning Participants were asked to rate items examining how they perceived the COVID-19 outbreak and how it has affected them. They rated measures of appreciation of life (four items; e.g., "This crisis has made me appreciate things I've taken for granted"), self-improvement motivations (four items; e.g., "This crisis has made me realize that I want to improve myself'), and complex beliefs about life (six items; e.g., "This crisis has increased the complexity of my beliefs about life") on seven-point scales from 1 ("Not at all") to 7 ("Very much").

Covariates Age, gender ( $1=$ "male," $0=$ "female"), education level $(1=$ "No school or some grade/primary school" to $11=$ "Advanced degree beyond a Master's Degree"), annual household income $(1=$ "Less than $\$ 10000$ " to $8=$ " $\$ 150,000$ or more"), and household size were assessed as demographical covariates. Eight items from the Balanced Inventory of Desirable Responding (BIDR-16; Hart et al., 2015) were administered to control for socially desirable tendencies, rated on a seven-point scale from 1 ("Strongly Disagree") to 7 ("Strongly Agree"). Four items were reverse-coded. Following Hart et al. (2015), each item was scored such that "6" or "7" were scored " 1 " while ratings below " 6 " were scored " 0. " The eight scores were summed.

Checks Two attention checks were administered to detect inattentive responses (e.g., "Maintaining good hygiene, but for this question select the option ' 2 ' to show that you are paying attention").

\section{Results}

Descriptive statistics and pairwise comparisons of sample differences are summarized in Table 1. Details on pairwise comparisons regarding key predictor and outcome variables are provided in Supplementary Analyses A. In Study 1A, mixed emotions were positively correlated with positive emotions $(r$ $=0.42, p<0.001)$ and negative emotions $(r=0.55, p<0.001)$. 
Table 1 Descriptive statistics for all key variables in Study $1 \mathrm{~A}$ and $1 \mathrm{~B}$

\begin{tabular}{|c|c|c|c|c|c|c|c|}
\hline & \multicolumn{3}{|c|}{ Study $1 \mathrm{~A}$} & \multicolumn{3}{|c|}{ Study 1B } & \multirow{2}{*}{$\begin{array}{l}\text { Exploratory pairwise } \\
\text { comparisons }\end{array}$} \\
\hline & $M$ & $S D$ & Range & $M$ & $S D$ & Range & \\
\hline Age & 43.07 & 12.87 & $\begin{array}{r}22 \text { to } \\
73\end{array}$ & 22.57 & 3.62 & $\begin{array}{r}18 \text { to } \\
53\end{array}$ & $\begin{array}{c}F(1,751)=956.54, p< \\
0.001\end{array}$ \\
\hline Gender & 0.46 & 0.50 & $\begin{array}{c}155 \\
\text { Mal- } \\
\text { es }\end{array}$ & 0.35 & 0.48 & $\begin{array}{c}144 \\
\text { Mal- } \\
\text { es }\end{array}$ & $\begin{array}{c}F(1,750)=9.25, p= \\
0.002\end{array}$ \\
\hline Education & 6.25 & 1.88 & 2 to 11 & 5.14 & 1.20 & 3 to 11 & $\begin{array}{c}F(1,751)=96.56, p< \\
0.001\end{array}$ \\
\hline Income & 4.54 & 1.74 & 1 to 8 & 3.51 & 2.21 & 1 to 8 & $\begin{array}{c}F(1,751)=45.78, p< \\
0.001\end{array}$ \\
\hline Household size & 2.59 & 1.40 & 0 to 8 & 4.26 & 1.19 & 1 to 9 & $\begin{array}{c}F(1,751)=311.55, p< \\
0.001\end{array}$ \\
\hline Social desirability & 3.48 & 2.57 & 0 to 8 & 1.61 & 1.72 & 0 to 8 & $\begin{array}{c}F(1,751)=143.95, p< \\
0.001\end{array}$ \\
\hline Positive emotions & $\begin{array}{c}2.97 \\
(0.9- \\
4)\end{array}$ & 1.21 & $\begin{array}{l}1 \text { to } \\
6.71\end{array}$ & $\begin{array}{c}3.03 \\
(0.9- \\
3)\end{array}$ & 1.01 & 1 to 5.9 & $\begin{array}{c}F(1,665)=0.98, p= \\
0.32\end{array}$ \\
\hline Negative emotions & $\begin{array}{c}2.76 \\
(0.9- \\
3)\end{array}$ & 1.14 & 1 to 5.8 & $\begin{array}{c}3.01 \\
(0.9- \\
0)\end{array}$ & 0.93 & 1 to 7 & $\begin{array}{c}F(1,665)=1.35, p= \\
0.25\end{array}$ \\
\hline Mixed emotions & $\begin{array}{c}3.02 \\
(0.9- \\
6)\end{array}$ & 1.21 & 1 to 7 & $\begin{array}{c}3.13 \\
(0.9- \\
5)\end{array}$ & 1.09 & 1 to 7 & $\begin{array}{c}F(1,665)=0.17, p= \\
0.68\end{array}$ \\
\hline $\begin{array}{l}\text { Legitimate } \\
\text { virus-prevention }\end{array}$ & $\begin{array}{c}5.46 \\
(0.7- \\
9)\end{array}$ & 1.22 & 1 to 7 & $\begin{array}{c}4.95 \\
(0.7- \\
8)\end{array}$ & 1.23 & $\begin{array}{c}1.33 \text { to } \\
7\end{array}$ & $\begin{array}{c}F(1,665)=7.84, p= \\
0.005\end{array}$ \\
\hline $\begin{array}{l}\text { Unsupported } \\
\text { virus-prevention }\end{array}$ & $\begin{array}{c}2.60 \\
(0.8- \\
3)\end{array}$ & 1.41 & $\begin{array}{l}1 \text { to } \\
6.67\end{array}$ & $\begin{array}{c}2.42 \\
(0.7- \\
8)\end{array}$ & 1.10 & 1 to 7 & $\begin{array}{c}F(1,665)=12.70, p< \\
0.001\end{array}$ \\
\hline $\begin{array}{l}\text { Atypical } \\
\text { virus-prevention }\end{array}$ & $\begin{array}{c}1.77 \\
(0.7- \\
8)\end{array}$ & 1.05 & 1 to 7 & $\begin{array}{c}1.45 \\
(0.7- \\
2)\end{array}$ & 0.62 & 1 to 4.5 & $\begin{array}{c}F(1,665)=62.51, p< \\
0.001\end{array}$ \\
\hline Appreciation & $\begin{array}{c}4.85 \\
(0.9- \\
6)\end{array}$ & 1.84 & 1 to 7 & $\begin{array}{c}5.18 \\
(0.9- \\
5)\end{array}$ & 1.49 & 1 to 7 & $\begin{array}{c}F(1,623)=3.59, p= \\
0.059\end{array}$ \\
\hline Self-improvement & $\begin{array}{c}4.35 \\
(0.9- \\
6)\end{array}$ & 1.92 & 1 to 7 & $\begin{array}{c}4.11 \\
(0.9- \\
4)\end{array}$ & 1.65 & 1 to 7 & $\begin{array}{c}F(1,620)=0.48, p= \\
0.49\end{array}$ \\
\hline Complex beliefs & $\begin{array}{c}4.34 \\
(0.9- \\
4)\end{array}$ & 1.69 & 1 to 7 & $\begin{array}{c}5.00 \\
(0.9- \\
1)\end{array}$ & 1.39 & 1 to 7 & $\begin{array}{c}F(1,622)=11.35, p= \\
0.001\end{array}$ \\
\hline
\end{tabular}

Parentheses beside the means indicate Cronbach's alpha for the variable and study. Gender was coded with $0=$ female and $1=$ male, and mean scores hence indicate gender proportions. Exploratory pairwise comparisons indicate whether mean scores for each variable differ between the two samples. This was done using ANOVAs for demographic comparisons, while pairwise comparisons for key predictors and outcomes were performed using ANCOVAs to adjust for demographic differences. The latter comparisons are presented in Supplementary Analyses A in more detail
Similarly, in Study 1B, mixed emotions were also positively correlated with positive emotions $(r=0.66, p<0.001)$ and negative emotions $(r=0.61, p<0.001)$. Positive and negative emotions were not correlated in Study 1A $(r=-0.05, p=0.36)$ but were positively correlated in Study 1B $(r=0.31, p<$ 0.001). As noted by Dejonchkheere et al. (2019), it is hence important to adjust for positive and negative emotions in order to conclude that any associations are independent of single- valenced emotions. Analyses were performed using $R$ (lavaan package) to conduct latent variable structural equation modelling. Missing data was addressed using full-information maximum likelihood (FIML) procedures, which are gold-standard procedures for handling missing data (Enders \& Bandalos, 2001).

We first tested the measurement model using confirmatory factor analyses in Study 1A and 1B. Randomized 
parceling was conducted following recommendations of Landis et al. (2000) to reduce the number of indicators per latent factor. Items measuring positive, negative, and mixed emotions were respectively randomly assigned into five parcels each, while items measuring legitimate, unsupported, and atypical behaviors were respectively randomly assigned into three parcels each. Items measuring complex beliefs about life were randomly assigned into three parcels, while items measuring appreciation of life and self-improvement motivations were specified directly as indicators. Overall eudaimonic functioning was specified as a higher-order factor comprising the lower-order factors of appreciation of life, self-improvement motivations, and complex beliefs about life. Model fit was strong in Study 1A, $\chi^{2}(536)=977.48, p<0.001, \mathrm{CFI}=0.96$, RMSEA $=0.049$, SRMR $=0.050$, and $1 \mathrm{~B}, \chi^{2}(536)=$ 931.79, $p<0.001, \mathrm{CFI}=0.97$, RMSEA $=0.042$, SRMR $=0.044$.

Next, we tested the structural models for both studies. We examined whether mixed emotions predicted legitimate, unsupported, and atypical virus-prevention behaviors, as well as overall eudaimonic functioning, controlling for the latent factors of positive emotions, negative emotions, and the observed variables for age, gender, education, income, household size, and social desirability. All predictors were allowed to covary. Model fit for the structural model was strong in both Study $1 \mathrm{~A}, \chi^{2}(704)=$ $1194.72, p<0.001, \mathrm{CFI}=0.96$, RMSEA $=0.045$, SRMR $=0.047$, and $1 \mathrm{~B}, \chi^{2}(704)=1150.74, p<0.001, \mathrm{CFI}=$ 0.96, RMSEA $=0.039, \mathrm{SRMR}=0.042$. The measurement and structural models of Study 1A and 1B are provided in Supplementary Figs. 1 and 2 respectively.

As summarized in Table 2, in Study 1A, mixed emotions predicted higher legitimate virus-prevention behaviors and eudaimonic functioning, whereas positive emotions and negative emotions did not predict these outcomes. Mixed emotions also predicted higher endorsements of unsupported virus-prevention behaviors but did not predict atypical virus-prevention behaviors. In contrast, positive emotions and negative emotions both predicted higher endorsements of unsupported and atypical virus-prevention behaviors. Key findings from Study 1B were largely consistent with Study 1A, such that mixed emotions significantly predicted legitimate virusprevention behaviors and higher eudaimonic functioning, but not atypical virus-prevention behaviors. However, mixed emotions did not predict unsupported virusprevention behaviors in Study 1B. Positive emotions did not predict any of the outcomes in Study 1B, while negative emotions predicted higher endorsements of unsupported virus-prevention behaviors and atypical virusprevention behaviors as well as lower eudaimonic functioning.

\section{Study 2}

Study 2 was conducted in the USA from $3^{\text {rd }}$ April (277,205 infections) to $5^{\text {th }}$ April $(330,891$ infections) and aimed to replicate the key results of Study 1. Study 2 also tested the theoretical model in which receptivity may mediate the associations of mixed emotions.

\section{Method}

\section{Participants}

As per Study 1, we aimed to collect at least 300 participants. We recruited 318 participants from the USA who had at least a 99\% approval rate and 10,000 HITs completed via MTurk. They were each reimbursed with USD \$1.00. Eight participants failed attention checks and were excluded. The final sample consisted of 310 participants (see Table 3 for demographic details). As per Study 1, participants were given a full debriefing after the study (Appendix C). Data and $R$ codes are available at https://osf.io/tbwc5/?view_only= 2cc3ae50f0f24f91ba7d206b21381d15.

\section{Measures}

The same items measuring emotions, virus-prevention behaviors, eudaimonic functioning, attention checks, and covariates from Study 1 were used. In addition, Study 2 included three receptivity items $(\alpha=0.78)$ that assessed how open participants were to new ways of coping with the crisis ("I am receptive to new ways of looking at the current crisis.", "I am open to trying new ways to cope to avoid getting infected.", "If I am infected, I would be open to trying new ways to be cured of the infection.") on a 7-point scale from 1 ("Strongly Disagree") to 7 ("Strongly Agree"). Cronbach's alphas for all measures are presented in Table 3. Materials are provided in Appendix A.

\section{Results}

Descriptive statistics are summarized in Table 3, and exploratory descriptive findings regarding whether the worsening crisis (operationalized as the number of infection cases on the day of participation) correlated with the variables when combining Study 1A and Study 2 (US samples collected about a week apart) are reported in Supplementary Analyses A. Mixed emotions were positively correlated with positive emotions $(r=0.37, p<$ $0.001)$ and negative emotions $(r=0.46, p<0.001)$, while positive emotions were negatively correlated with negative emotions $(r=-0.28, p<0.001)$. Analyses were performed using the same procedures in Study 1. Model fit was strong for both the measurement model, $\chi^{2}(536)=953.50, p<$ 
Table 2 Standardized latent variable path coefficients predicting all outcome variables in Study 1A and 1B

\begin{tabular}{|c|c|c|c|c|c|c|c|c|c|c|c|c|}
\hline \multirow[b]{2}{*}{ Study $1 \mathrm{~A}$} & \multicolumn{3}{|c|}{ Legitimate behaviors } & \multicolumn{3}{|c|}{ Unsupported behaviors } & \multicolumn{3}{|c|}{ Atypical behaviors } & \multicolumn{3}{|c|}{ Eudaimonic functioning } \\
\hline & $\beta$ & $p$ & $95 \% \mathrm{CI}$ & $\beta$ & $p$ & $95 \% \mathrm{CI}$ & $\beta$ & $p$ & $95 \% \mathrm{CI}$ & $\beta$ & $p$ & $95 \% \mathrm{CI}$ \\
\hline Age & 0.03 & 0.62 & {$[-0.015,0.01]$} & 0.14 & 0.013 & {$[-0.03,-0.003]$} & -0.19 & 0.001 & {$[-0.02,-0.01]$} & -0.01 & 0.80 & {$[-0.02,0.01]$} \\
\hline Gender & -0.13 & 0.028 & {$[-0.62,-0.04]$} & -0.02 & 0.74 & {$[-0.32,0.23]$} & 0.09 & 0.099 & {$[-0.03,0.37]$} & -0.11 & 0.026 & {$[-0.69,-0.04]$} \\
\hline Education & 0.03 & 0.57 & {$[-0.06,0.11]$} & 0.01 & 0.85 & {$[-0.07,0.09]$} & 0.03 & 0.57 & {$[-0.04,0.07]$} & 0.02 & 0.71 & {$[-0.07,0.11]$} \\
\hline Income & 0.06 & 0.35 & {$[-0.05,0.14]$} & -0.06 & 0.32 & {$[-0.14,0.05]$} & -0.17 & 0.005 & {$[-0.16,-0.03]$} & 0.03 & 0.60 & {$[-0.08,0.14]$} \\
\hline HH size & 0.10 & 0.12 & {$[-0.02,0.21]$} & 0.14 & 0.013 & {$[0.03,0.24]$} & 0.21 & $<0.001$ & {$[0.06,0.22]$} & 0.09 & 0.13 & {$[-0.03,0.22]$} \\
\hline S. Des & 0.31 & $<0.001$ & {$[0.10,0.22]$} & 0.10 & 0.071 & {$[-0.004,0.11]$} & -0.03 & 0.65 & {$[-0.05,0.03]$} & 0.14 & 0.006 & {$[0.03,0.16]$} \\
\hline Pos. E & -0.11 & 0.14 & {$[-0.30,0.04]$} & 0.22 & 0.001 & {$[0.11,0.43]$} & 0.26 & $<0.001$ & {$[0.11,0.35]$} & 0.09 & 0.17 & {$[-0.06,0.32]$} \\
\hline Neg. E & 0.10 & 0.24 & {$[-0.08,0.32]$} & 0.18 & 0.023 & {$[0.03,0.41]$} & 0.32 & $<0.001$ & {$[0.14,0.41]$} & -0.12 & 0.12 & {$[-0.40,0.04]$} \\
\hline Mix. E & 0.34 & $<0.001$ & {$[0.17,0.59]$} & 0.29 & 0.001 & {$[0.14,0.53]$} & 0.11 & 0.22 & {$[-0.05,0.23]$} & 0.58 & $<0.001$ & {$[0.55,1.02]$} \\
\hline Study 1B & $\beta$ & $p$ & $95 \% \mathrm{CI}$ & $\beta$ & $p$ & $95 \% \mathrm{CI}$ & $\beta$ & $p$ & $95 \% \mathrm{CI}$ & $\beta$ & $p$ & $95 \% \mathrm{CI}$ \\
\hline Age & 0.04 & 0.44 & {$[-0.02,0.05]$} & 0.08 & 0.15 & {$[-0.01,0.06]$} & -0.01 & 0.91 & {$[-0.02,0.01]$} & 0.01 & 0.85 & {$[-0.03,0.04]$} \\
\hline Gender & -0.10 & 0.073 & {$[-0.47,0.02]$} & -0.04 & 0.51 & {$[-0.31,0.15]$} & -0.07 & 0.27 & {$[-0.16,0.04]$} & 0.01 & 0.90 & {$[-0.21,0.24]$} \\
\hline Education & -0.03 & 0.57 & {$[-0.13,0.07]$} & 0.05 & 0.40 & {$[-0.05,0.14]$} & -0.01 & 0.90 & {$[-0.04,0.04]$} & 0.01 & 0.84 & {$[-0.09,0.10]$} \\
\hline Income & 0.02 & 0.71 & {$[-0.05,0.07]$} & -0.04 & 0.54 & {$[-0.07,0.04]$} & -0.09 & 0.17 & {$[-0.04,0.01]$} & 0.09 & 0.09 & {$[-0.01,0.10]$} \\
\hline HH size & -0.09 & 0.098 & {$[-0.18,0.02]$} & 0.02 & 0.70 & {$[-0.07,0.11]$} & 0.02 & 0.71 & {$[-0.03,0.05]$} & -0.02 & 0.77 & {$[-0.10,0.08]$} \\
\hline S. Des & 0.15 & 0.005 & {$[0.03,0.16]$} & -0.09 & 0.073 & {$[-0.12,0.01]$} & 0.00 & 0.97 & {$[-0.03,0.03]$} & 0.08 & 0.099 & {$[-0.01,0.11]$} \\
\hline Pos. E & -0.09 & 0.29 & {$[-0.29,0.09]$} & 0.06 & 0.46 & {$[-0.11,0.24]$} & 0.04 & 0.65 & {$[-0.06,0.09]$} & 0.09 & 0.24 & {$[-0.07,0.28]$} \\
\hline Neg. E & 0.11 & 0.15 & {$[-0.06,0.38]$} & 0.24 & 0.003 & {$[0.11,0.53]$} & 0.23 & 0.016 & {$[0.02,0.21]$} & -0.16 & 0.023 & {$[-0.44,-0.03]$} \\
\hline Mix. E & 0.33 & 0.002 & {$[0.12,0.54]$} & 0.11 & 0.30 & {$[-0.09,0.30]$} & -0.02 & 0.84 & {$[-0.09,0.08]$} & 0.61 & $<0.001$ & {$[0.42,0.82]$} \\
\hline
\end{tabular}

$\mathrm{HH}$ size = household size; $\mathrm{S}$. Des = social desirability; Pos. $\mathrm{E}=$ positive emotions; Neg. $\mathrm{E}=$ negative emotions; Mix. $\mathrm{E}=$ mixed emotions

$0.001, \mathrm{CFI}=0.96, \mathrm{RMSEA}=0.050, \mathrm{SRMR}=0.049$, and the structural model, $\chi^{2}(704)=1223.88, p<0.001, \mathrm{CFI}=$

Table 3 Descriptive statistics for all key variables in Study 2

\begin{tabular}{llll}
\hline & $M$ & $S D$ & Range \\
\hline Age & 42.63 & 12.74 & 22 to 79 \\
Gender & 0.39 & 0.49 & 119 Males \\
Education & 6.57 & 1.86 & 2 to 11 \\
Income & 4.84 & 1.72 & 1 to 8 \\
Household size & 2.55 & 1.35 & 0 to 8 \\
Social desirability & 3.52 & 2.49 & 0 to 8 \\
Positive emotions & $3.20(0.94)$ & 1.19 & 1 to 6.71 \\
Negative emotions & $2.92(0.93)$ & 1.13 & 1 to 6.25 \\
Mixed emotions & $3.18(0.96)$ & 1.21 & 1 to 6.36 \\
Legitimate virus-prevention & $5.80(0.78)$ & 1.07 & 1 to 7 \\
Unsupported virus-prevention & $2.95(0.84)$ & 1.45 & 1 to 7 \\
Atypical virus-prevention & $1.82(0.75)$ & 1.02 & 1 to 6.17 \\
Appreciation & $5.22(0.96)$ & 1.66 & 1 to 7 \\
Self-improvement & $4.63(0.97)$ & 1.77 & 1 to 7 \\
Complex beliefs & $4.46(0.95)$ & 1.66 & 1 to 7 \\
Receptivity & $5.38(0.78)$ & 1.20 & 1.33 to 7 \\
\hline
\end{tabular}

Parentheses beside the means indicate Cronbach's alpha
0.95, RMSEA $=0.049$, SRMR $=0.047$. The measurement and structural model is provided in Supplementary Fig. 3. Results largely replicated Study 1, such that mixed emotions predicted legitimate virus-prevention behaviors and eudaimonic functioning but not atypical virus-prevention behaviors (Table 4). The finding that mixed emotions predicted unsupported virus-prevention behaviors in Study $1 \mathrm{~A}$ was not replicated. Neither positive emotions nor negative emotions predicted legitimate virus-prevention behaviors but both predicted endorsements of unsupported and atypical virus-prevention behaviors, consistent with Study 1A. Negative emotions did not predict eudaimonic functioning, while positive emotions predicted higher eudaimonic functioning.

Next, we examined the mediational model. All model specifications are identical to the above, except the addition of receptivity as a latent variable indicated by three items. Receptivity was specified as an outcome variable predicted by mixed emotions, controlling for positive emotions, negative emotions, demographics, and social desirability. Additionally, receptivity was specified as a predictor of legitimate virus-prevention behaviors and eudaimonic functioning. Analyses were bootstrapped with 10,000 resamples, and the indirect effects of mixed emotions via receptivity were computed. Model fit was strong 
Table 4 Standardized latent variable path coefficients predicting all outcome variables in Study 2

\begin{tabular}{|c|c|c|c|c|c|c|c|c|c|c|c|c|}
\hline & \multicolumn{3}{|c|}{ Legitimate behaviors } & \multicolumn{3}{|c|}{ Unsupported behaviors } & \multicolumn{3}{|c|}{ Atypical behaviors } & \multicolumn{3}{|c|}{ Eudaimonic functioning } \\
\hline & $\beta$ & $p$ & $95 \% \mathrm{CI}$ & $\beta$ & $p$ & $95 \% \mathrm{CI}$ & $\beta$ & $p$ & $95 \% \mathrm{CI}$ & $\beta$ & $p$ & $95 \% \mathrm{CI}$ \\
\hline Age & -0.02 & 0.77 & {$[-0.01,0.01]$} & -0.02 & 0.76 & {$[-0.02,0.01]$} & -0.11 & 0.070 & {$[-0.01,0.001]$} & -0.05 & 0.36 & {$[-0.02,0.01]$} \\
\hline Gender & -0.12 & 0.058 & {$[-0.57,0.01]$} & -0.14 & 0.016 & {$[-0.79,-0.08]$} & 0.02 & 0.70 & {$[-0.15,0.22]$} & -0.11 & 0.028 & {$[-0.59,-0.03]$} \\
\hline Education & -0.09 & 0.18 & {$[-0.13,0.03]$} & -0.01 & 0.82 & {$[-0.11,0.09]$} & -0.03 & 0.66 & {$[-0.06,0.04]$} & -0.10 & 0.060 & {$[-0.15,0.003]$} \\
\hline Income & 0.07 & 0.33 & {$[-0.05,0.14]$} & -0.12 & 0.064 & {$[-0.22,0.01]$} & -0.24 & $<0.001$ & {$[-0.17,-0.05]$} & 0.10 & 0.071 & {$[-0.01,0.17]$} \\
\hline HH Size & 0.14 & 0.034 & {$[0.01,0.23]$} & 0.12 & 0.062 & {$[-0.01,0.27]$} & 0.06 & 0.35 & {$[-0.04,0.11]$} & 0.07 & 0.18 & {$[-0.03,0.18]$} \\
\hline S. Des & 0.03 & 0.64 & {$[-0.05,0.08]$} & -0.02 & 0.71 & {$[-0.09,0.06]$} & -0.19 & 0.004 & {$[-0.10,-0.02]$} & 0.19 & 0.001 & {$[0.05,0.16]$} \\
\hline Pos. E & -0.08 & 0.38 & {$[-0.28,0.11]$} & 0.33 & $<0.001$ & {$[0.22,0.70]$} & 0.33 & $<0.001$ & {$[0.11,0.37]$} & 0.25 & 0.001 & {$[0.14,0.51]$} \\
\hline Neg. E & -0.04 & 0.68 & {$[-0.27,0.18]$} & 0.25 & 0.007 & {$[0.10,0.66]$} & 0.26 & 0.006 & {$[0.06,0.36]$} & 0.03 & 0.71 & {$[-0.18,0.26]$} \\
\hline Mix. E & 0.29 & 0.004 & {$[0.09,0.47]$} & 0.03 & 0.78 & {$[-0.20,0.27]$} & 0.04 & 0.65 & {$[-0.10,0.15]$} & 0.43 & $<0.001$ & {$[0.32,0.69]$} \\
\hline
\end{tabular}

$\mathrm{HH}$ size = household size; S. Des = social desirability; Pos. $\mathrm{E}=$ positive emotions; Neg. $\mathrm{E}=$ negative emotions; Mix. $\mathrm{E}=$ mixed emotions

for both the measurement model, $\chi^{2}(634)=1125.00, p<$ $0.001, \mathrm{CFI}=0.96, \mathrm{RMSEA}=0.050, \mathrm{SRMR}=0.053$, and the structural model, $\chi^{2}(832)=1462.67, p<0.001, \mathrm{CFI}=$ 0.95, RMSEA $=0.050, \mathrm{SRMR}=0.057$. The measurement and structural model for the mediation is depicted in Supplementary Fig. 4, while the summarized figure of the mediational model is provided in Fig. 1.

Results indicated that mixed emotions predicted higher receptivity $(\beta=0.40, p<0.001,95 \% \mathrm{CI}[0.18,0.63])$ and receptivity in turn predicted higher virus-prevention behaviors $(\beta=0.56, p<0.001,95 \%$ CI $[0.35,0.76])$ and eudaimonic functioning $(\beta=0.46, p<0.001,95 \% \mathrm{CI}$ $[0.35,0.74]$. The indirect effects of mixed emotions via receptivity on legitimate virus-prevention behaviors (indirect effect $=0.22,95 \% \mathrm{CI}[0.08,0.39]$ ) and eudaimonic functioning (indirect effect $=0.21,95 \%$ CI $[0.09,0.37]$ ) were significant. Alternative mediational models (see Supplementary Fig. 5) in which mixed emotions were instead specified as mediators between receptivity and legitimate virus-prevention behaviors or eudaimonic functioning received less support. Specifically, while the alternative mediational model was also supported for eudaimonic functioning, indirect effect $=0.06,95 \%$ CI $[0.02,0.11]$, there was no support for this model for legitimate virusprevention behaviors, indirect effect $=0.01,95 \% \mathrm{CI}$ $[-0.04,0.06]$. The theoretical model in which receptivity is specified as the mediator is hence more consistently supported across both outcome variables.

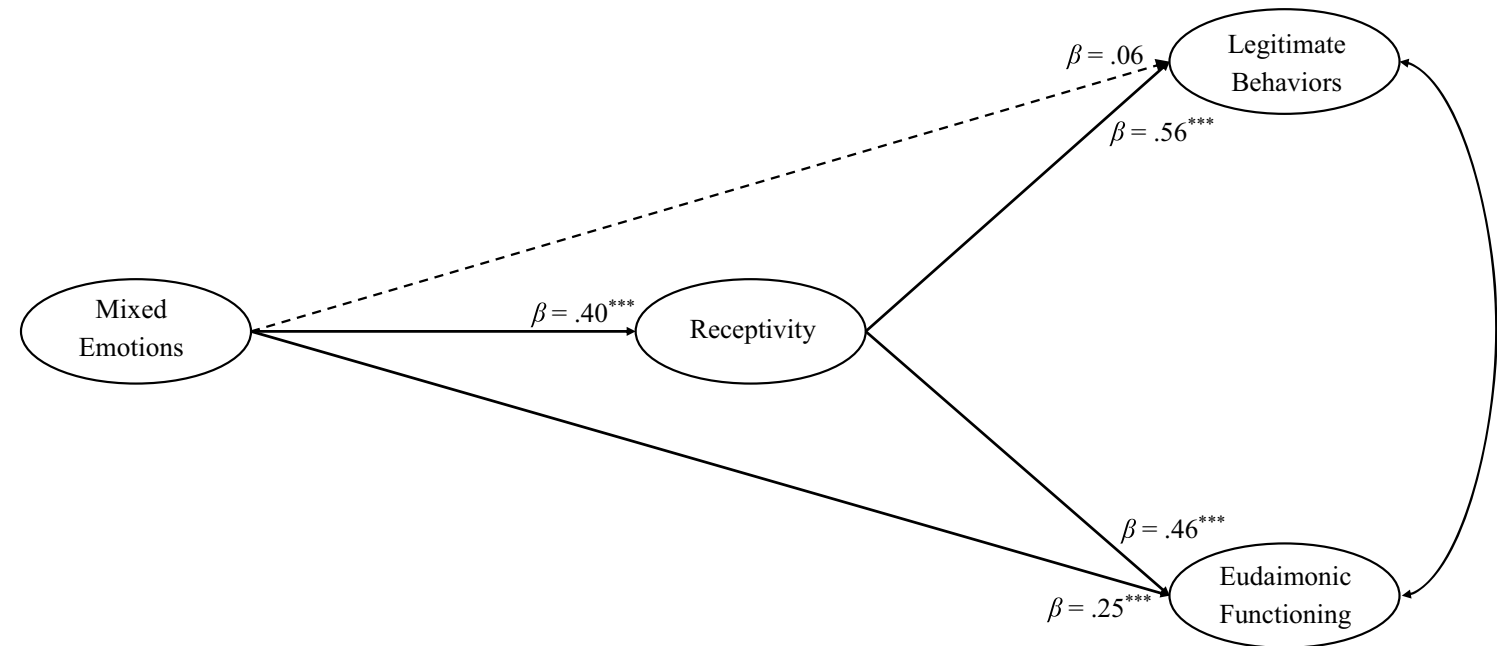

Fig. 1 Mediational model of mixed emotions predicting legitimate virusprevention behaviors and eudaimonic functioning via receptivity in Study 2 . Bolded lines represent significant paths while dashed lines indicate nonsignificant paths. ${ }^{* * *} p<0.001$. Positive emotions, negative emotions, age, gender, education level, household income, household size, and social desirability were controlled for in all pathways. Indirect effects of mixed emotions via receptivity were significant for both legitimate virusprevention behaviors (indirect effect $=0.22,95 \%$ CI $[0.08,0.39]$ ) and eudaimonic functioning (indirect effect $=0.21,95 \%$ CI $[0.09,0.37]$ ) 


\section{Study 3}

Study 3 provided a preregistered experimental study to test whether incidental mixed emotions experienced in the moment (e.g., Lerner \& Keltner, 2001) would influence immediate intentions to enact legitimate virusprevention behaviors and eudaimonic functioning relative to positive emotion, negative emotion, and neutral control groups. Study 3 was conducted between 9 April $(459,165$ infections) and 20 April (776,093 infections). As mixed emotions were largely unrelated to unsupported and atypical virus-prevention behaviors in Study 1 and Study 2, these outcomes were not assessed in Study 3. Additionally, as the causal relationship between mixed emotions and receptivity has previously been established (Rees et al., 2013), we did not further examine receptivity in Study 3. These omissions keep the study shorter and reduce the likelihood that the elicited emotions would dissipate due to fatigue while participants are completing the outcome measures. We hence focused on replicating the main findings of Study 1 and Study 2, which revealed consistently positive associations between mixed emotions with legitimate virus-prevention behaviors and eudaimonic functioning. We hypothesized that mixed emotions would lead to higher endorsements of these two outcomes relative to other conditions.

\section{Method}

\section{Participants}

Study 3 was preregistered at http://aspredicted.org/blind.php? $\mathrm{x}=\mathrm{i} 8 \mathrm{ni5t}$ t. Assuming a medium effect size based on Study 1 and Study 2, power analyses for a four-condition betweensubjects experiment indicated a minimum $N=179$ for a power of 0.80 and alpha of 0.05 . As experimental effect sizes may be attenuated by data noise due to online experimental settings, we sought to collect at least 300 participants after exclusions, which would allow for 0.80 power even if effect sizes were small to medium. Based on previous MTurk experimental studies which usually exclude between 15 and $25 \%$ of participants (e.g., Young et al., 2018), we took a conservative approach and projected up to a $30 \%$ exclusion rate, given that more extensive exclusion criteria are used in the present study. Hence, we aimed to collect at least 430 participants to allow for 300 participants even if $30 \%$ of participants were excluded. We recruited 437 US participants via Amazon MTurk. Participants were reimbursed USD \$1.00. After exclusions (see the "Data Quality Checks" section), the final $N$ was 358 (155 males, 201 females, 2 others; $M_{\text {age }}=43.15, S D_{\text {age }}=12$. 41, age range: $21-88$ years). Data is available at https://osf.io/ tbwc5/?view_only=2cc3ae50f0f24f91ba7d206b21381d15.

\section{Materials}

A smaller set of items from Study 1 and Study 2 were used to keep the study short and to ensure that the manipulated emotions remained active while participants completed the dependent measures. Items which maintained a balance between high item-total correlations, factor loadings, and variability as assessed based on means and standard deviation scores were selected (see Appendix A for more details). The shortened scales maintained very strong correlations with the full scales ( $r=0.93$ for legitimate virus-prevention behaviors and $r$ $=0.95$ for eudaimonic functioning) and are thus likely to reliably capture the same construct. Three items (e.g., "Avoid touching your face.") assessed legitimate virus-prevention behaviors and four items (e.g., "This crisis has increased how much I value the little things in life.") assessed eudaimonic functioning. For manipulation checks, participants rated how they felt during the recall task on seven positive emotion items, seven negative emotion items, and five mixed emotion items. In addition, participants completed data quality and hypothesis check items. Cronbach's alphas are presented in Table 5.

\section{Procedure}

Participants were randomly assigned to the following conditions: (1) mixed emotions; (2) positive emotions; (3) negative emotions; and (4) neutral (see full instructions in Appendix B). Participants in the emotion conditions were asked to think about how the COVID-19 crisis has impacted them and caused them to feel either (1) positive emotions and negative emotions at the same time; (2) positive emotions; or (3) negative emotions. Participants in the neutral condition described their daily morning routine. All participants were instructed to spend 5 minutes describing their experiences. Next, participants completed legitimate virus-prevention behavior items. Next, as emotion manipulations are transient, we instructed participants to undergo another round of emotion manipulation, where they reflected on the description they provided previously and wrote a few more lines to strengthen the manipulation. Next, the measure of eudaimonic functioning was administered. Finally, participants completed manipulation, data quality, and hypothesis-awareness check items. Participants were then given a full debriefing (Appendix C).

\section{Data Quality Checks}

As online experiments have lower experimental control, we employed several approaches to maximize the reliability of our data. One approach was through data quality and hypothesis-awareness items. Prior to the experiment, participants completed an attention check where they were told to indicate the option "negative two" on a seven- 
Table 5 Cell means for manipulation checks and outcome variables in Study 3

\begin{tabular}{|c|c|c|c|c|c|c|c|c|}
\hline & \multicolumn{2}{|c|}{$\begin{array}{l}\text { Mixed emotion } \\
\text { condition }\end{array}$} & \multicolumn{2}{|c|}{$\begin{array}{l}\text { Positive } \\
\text { emotion } \\
\text { condition }\end{array}$} & \multicolumn{2}{|c|}{$\begin{array}{l}\text { Negative } \\
\text { emotion } \\
\text { condition }\end{array}$} & \multicolumn{2}{|c|}{$\begin{array}{l}\text { Neutral } \\
\text { condition }\end{array}$} \\
\hline & $M$ & $S D$ & $M$ & $S D$ & $M$ & $S D$ & $M$ & $S D$ \\
\hline Positive emotions $(\alpha=0.91)$ & $3.64^{\mathrm{bc}}$ & 1.21 & $4.49^{\text {acd }}$ & 1.27 & $2.76^{\mathrm{abd}}$ & 1.38 & $3.59^{\mathrm{bc}}$ & 1.43 \\
\hline Negative emotions $(\alpha=0.92)$ & $3.58^{\mathrm{bcd}}$ & 1.39 & $2.40^{\mathrm{ac}}$ & 1.25 & $4.37^{\mathrm{abd}}$ & 1.27 & $2.14^{\mathrm{ac}}$ & 1.37 \\
\hline Mixed emotions $(\alpha=0.93)$ & $4.76^{\mathrm{bcd}}$ & 1.25 & $3.75^{\mathrm{ad}}$ & 1.42 & $3.38^{\mathrm{ad}}$ & 1.62 & $2.90^{\mathrm{abc}}$ & 1.49 \\
\hline Legitimate behaviors ( $\alpha=0.66$ ) & $6.22^{\mathrm{bcd}}$ & 0.72 & $5.80^{\mathrm{a}}$ & 1.06 & $5.87^{\mathrm{a}}$ & 1.29 & $5.60^{\mathrm{a}}$ & 1.19 \\
\hline Eudaimonic functioning ( $\alpha=0.96$ ) & $5.63^{\mathrm{d}}$ & 1.12 & $5.41^{\mathrm{d}}$ & 1.20 & $5.61^{\mathrm{d}}$ & 1.19 & $4.96^{\mathrm{abc}}$ & 1.35 \\
\hline
\end{tabular}

Cell mean is significantly different from the mixed emotion condition; ${ }^{\mathrm{b}}$ cell mean is significantly different from the positive emotion condition; ${ }^{\mathrm{c}}$ cell mean is significantly different from the negative emotion condition; ${ }^{\mathrm{d}}$ cell mean is significantly different from the neutral condition point scale (from -3 to 3 ); participants who failed this check were automatically disqualified from further participation. At the end of the experiment, participants reported whether they completed the study in a private room; talked to someone during the study; and were interrupted during the study. Online studies lack the advantage of laboratory studies where participants could focus on the study diligently and greater experimental control could be achieved. These measures assess whether the current participants completed the study in conditions similar to isolated laboratory-based settings. We excluded 4 participants who reported not being in a private room, 7 who reported talking to someone, and 5 who reported being interrupted. Moreover, 13 participants were excluded for discerning the purpose of the experiment.

Additionally, several other approaches were taken as well. One participant was flagged by Qualtrics to be a bot, and 36 participants did not comply with the emotion recall task instructions and provided descriptions that were inappropriate for their assigned conditions. Using duration metrics provided by Qualtrics, we identified 11 participants whose duration of participation exceeded the 97.5th percentile. We employed TaskMaster (Permut et al., 2019) to assess the duration participants spent outside the study page (i.e., looking at other webpages), and we identified 11 participants who had outside-study duration that exceeded the 97.5th percentile. To account for individual variability in response times and to retain as many participants as possible (Curran, 2016), we used a conservative estimate to exclude only the most extreme outliers who were above the 97.5th percentile on these two metrics. All these participants were excluded. In sum, 79 participants $(18.1 \%)$ were excluded (some participants failed more than one check and hence the numbers did not sum to exactly 79). This exclusion rate is consistent with other online experimental studies (e.g., Young et al., 2018).

\section{Results}

\section{Manipulation Checks}

One-way ANOVAs indicate significant differences between conditions in positive emotions, $F(3,354)=25.02, p<0.001$, $\eta_{\mathrm{p}}{ }^{2}=0.18$, negative emotions, $F(3,354)=56.20, p<0.001$, $\eta_{\mathrm{p}}{ }^{2}=0.32$, and mixed emotions, $F(3,354)=26.34, p<0.001$, $\eta_{\mathrm{p}}^{2}=0.18$. Planned contrasts indicate that mixed emotions were higher in the mixed emotion condition than other conditions, $t(356)=7.83, p<0.001$; positive emotions were higher in the positive emotion condition than other conditions ${ }^{2}$, $t(356)=6.83, p<0.001 ;$ and negative emotions were higher in the negative emotion condition than other conditions, $t(356)$ $=9.69, p<0.001$ (see Table 5).

\section{Main Analyses}

Separate one-way ANOVAs indicated significant differences between conditions in legitimate virus-prevention behaviors, $F(3,354)=5.03, p=0.002, \eta_{\mathrm{p}}{ }^{2}=0.041$, and eudaimonic functioning, $F(3,354)=5.95, p=0.001, \eta_{\mathrm{p}}{ }^{2}=0.048$. Planned contrasts indicate that legitimate virus-prevention behaviors, $t(356)=3.44, p=0.001$, and eudaimonic functioning, $t(356)=2.02, p=0.044$, were higher in the mixed emotion condition than the other conditions (see Table 5). One-tailed pairwise comparisons found that legitimate virus-prevention behaviors were higher in the mixed emotion condition

\footnotetext{
${ }^{2}$ Contrary to expectation, positive emotions - which are part of mixed emotions - did not differ between the mixed emotion $\left(M_{\text {positive-emotions }}=\right.$ $3.64)$ and neutral $\left(M_{\text {positive-emotions }}=3.59\right)$ conditions, though this did not affect the key outcomes of interest. Inspecting the mean scores, positive emotions were likely elicited at a mild to moderate intensity in both conditions, suggesting that the neutral condition may inadvertently have elicited some positive affect, possibly because recalling one's morning routine could have restored a sense of normalcy amidst an uncertain pandemic. Alternative neutral conditions (e.g., recalling a mundane activity such as dishwashing) should be examined in future work.
} 
compared to the neutral, $t(179)=4.21, p<0.001,95 \% \mathrm{CI}$ $[0.33,0.91], d=0.63$, positive emotion, $t(170)=3.03, p=$ $0.002,95 \%$ CI $[0.15,0.69], d=0.46$, and negative emotion conditions, $t(177)=2.20, p=0.015,95 \%$ CI $[0.04,0.66], d=$ 0.33 . In contrast, legitimate virus-prevention behaviors did not differ between the positive emotion and neutral conditions, $t(177)=1.20, p=0.23,95 \%$ CI $[-0.13,0.54], d=0.18$, and also did not differ between the negative emotion and neutral conditions, $t(184)=1.52, p=0.13,95 \%$ CI $[-0.08,0.64], d=$ 0.22 .

One-tailed pairwise comparisons also indicated that eudaimonic functioning was higher in the mixed emotion condition compared to the neutral condition, $t(179)=3.61, p<$ $0.001,95 \%$ CI $[0.30,1.03], d=0.54$. However, eudaimonic functioning did not differ between the mixed emotion condition and the positive emotion condition, $t(170)=1.26, p=$ $0.10,95 \% \mathrm{CI}[-0.13,0.57], d=0.19$, or the negative emotion condition, $t(177)=0.12, p=0.45,95 \%$ CI $[-0.32,0.36], d=$ 0.02 . Compared to the neutral condition, eudaimonic functioning was also higher in the positive emotion condition, $t(177)=2.31, p=0.022,95 \%$ CI $[-0.07,0.82], d=0.35$, and the negative emotion condition, $t(184)=3.46, p=$ $0.001,95 \%$ CI $[0.28,1.01], d=0.51$.

\section{General Discussion}

At a time when the COVID-19 pandemic was exponentially worsening, we found that mixed emotions were associated with self-reported intentions to enact legitimate virusprevention behaviors such as social distancing and good hygiene, and eudaimonic functioning involving appreciation, self-improvement, and developing complex insights about life, supporting the integrative perspective. These findings held in a comparison sample (Study 1A and 1B) and were replicated, with some evidence that receptivity may mediate these findings (Study 2). ${ }^{3}$ Furthermore, manipulated mixed emotions led to stronger intentions for enacting legitimate virus-prevention behaviors and eudaimonic functioning relative to a neutral condition (Study 3), although comparisons against positive and negative emotion conditions were significant only for virus-prevention behaviors but not eudaimonic functioning.

Conversely, positive and negative emotions were not linked to legitimate virus-prevention behaviors in any study, suggesting that positive associations are unique to mixed emotions. Moreover, mixed emotions were unrelated to atypical virus-prevention behaviors, while links with unsupported virus-prevention behaviors were found only in one study

\footnotetext{
${ }^{3}$ We performed additional analyses (reported in Supplementary Analyses B) in which no demographical covariates were included. The key findings remained consistent regardless of the inclusion of these covariates.
}

and were not replicable. Conversely, positive and negative emotions predicted unsupported and atypical virusprevention behaviors, which is consistent with findings that positive emotions may promote gullibility (Forgas \& East, 2008 ) and negative emotions may promote maladaptive coping (Tran et al., 2019). Links between eudaimonic functioning with positive and negative emotions were inconsistent, with both significant and nonsignificant associations across studies. In contrast, links between mixed emotions with eudaimonic functioning were largely consistent and replicable, though this was not differentiable from single-valenced emotions in Study 3.

These findings suggest that mixed emotions could have emotion-specific benefits for pandemic-related outcomes that are distinctive from positive and negative emotions. Specifically, mixed emotions may facilitate pandemicrelated psychological functioning and intentions of accepting scientific recommendations for protecting against COVID-19 (Lewnard \& Lo, 2020), and there is suggestive evidence that this may not generalize to the uncritical acceptance of misinformation. These findings are in line with research suggesting that mixed emotions facilitate integrative processes in which divergent information are utilized to enhance processing and coping outcomes (Rees et al., 2013) and suggest that the integrative perspective may be more strongly supported than the conflict perspective, at least within the context of an adversity like the pandemic (Larsen et al., 2003). In most analyses, the effect sizes of mixed emotions were medium to large (see Table 6 for a summary of effect sizes), suggesting potentially important associations that span both naturalistic and experimental designs. Moreover, despite many differences between the US and Singapore samples, we found preliminary evidence that processes related to mixed emotions were similar. Although some evidence suggests that mixed emotions primarily benefit East Asian cultures which are accepting of dialecticism (Miyamoto \& Ryff, 2011), their integrative benefits could be robust within the context of the pandemic. Nevertheless, rigorous cross-national examinations will be required to make clearer cross-cultural conclusions.

Several other important points bear mentioning. We examined mixed emotions using direct measures (Berrios et al., 2015), but indirect approaches such as the minimum index (MIN) are also viable indices of mixed emotions (Larsen et al., 2017). These approaches were not utilized in the present study as they more accurately capture simultaneous cooccurrence when state emotions in the moment are measured as opposed to emotions over a period of time. Further research to probe the strengths and limitations of using MIN or direct measures in different contexts would provide substantial methodological advancements. Additionally, despite some inconsistencies, social desirability tended to bias self-reports towards more favorable ratings, such as higher ratings of eudaimonic functioning and legitimate behaviors. Given the 
Table 6 Summary of the effect sizes of mixed emotions predicting legitimate virusprevention behaviors and eudaimonic functioning in all studies
Effect sizes based on standardized regression coefficient $(\beta)$

Legitimate virus-prevention behaviors

Eudaimonic functioning

\begin{tabular}{lcc}
\hline Study 1A & 0.34 & 0.58 \\
Study 1B & 0.33 & 0.61 \\
Study 2 & 0.29 & 0.43 \\
& Effect Sizes based on Cohen's $\boldsymbol{d}$ relative to neutral condition \\
Study 3 & 0.63 & 0.54 \\
\hline
\end{tabular}

As standardized regression coefficients are comparable to partial correlation coefficients, we interpreted these coefficients using conventions for the effect sizes of correlation coefficients. Based on Cohen (1988), $\beta=0.10$ and $d=0.20$ would correspond to a small effect size; $\beta=0.30$ and $d=0.50$ would correspond to a medium effect size; $\beta=0.50$ and $d=0.80$ would correspond to a large effect size. Effect sizes of mixed emotions hence generally fall between the medium to large range. Comparing these effects to other reported effect sizes in the field (Lakens, 2013), the effect sizes reported here are somewhat larger than the small-to-medium effect sizes that have been reported in some previous research (e.g., Berrios et al., 2018; Rees et al., 2013), though differences in methodology and the specific outcomes studied prevent direct comparisons strong emphasis on social responsibility in this pandemic, socially desirable tendencies may have strong biasing effects and may be important to adjust for in future pandemicrelated work (Timmons et al., 2020).

We also note several limitations. Firstly, as self-reported measures were used, the conclusions may not generalize to actual behaviors. Nevertheless, the self-reported measures can provide valuable insights into how emotions may influence behavioral intentions, which likely precede actual behaviors. Future work should also examine actual behaviors such as mask-wearing and other behavioral intentions such as the willingness to receive vaccinations for COVID-19. Secondly, Study 2 cannot conclusively establish whether receptivity mediated the associations of mixed emotions, or if mixed emotions instead mediated the effects of receptivity. However, as there is prior empirical evidence that mixed emotions causally elicited receptivity which in turn exerted a mediating effect (Rees et al., 2013), we sought to provide confirmatory support for this theory rather than establish causality. Finally, Study 3 did not support distinctions between mixed emotions and single-valenced emotions for eudaimonic functioning. One possibility is that the expressive writing task may encourage reappraisal processes independently of emotions (Baikie et al., 2012), leading all the emotion conditions to increase eudaimonic functioning. However, expressive writing specifically about mixed emotions had unique benefits on the virusprevention measure, which may be especially pertinent to public health outcomes, and other such unique benefits could have potential for further research.

Overall, we provide evidence that mixed emotions may have naturalistic and causal links with intentions to enact legitimate virus-prevention behaviors that could reduce infection rates and eudaimonic functioning amidst the COVID-19 pandemic. Mixed emotions may thus have theoretical implications over and above single-valenced emotions and may also have practical implications for important outcomes experienced during this crisis.

Supplementary Information The online version contains supplementary material available at https://doi.org/10.1007/s42761-021-00045-x.

Data Availability Data are publicly available at https://osf.io/tbwc5/? view_only=2cc3ae50f0f24f91ba7d206b21381d15.

\section{Additional Information}

Conflicts of Interest The authors declare no competing interests.

Ethics Approval The study was approved by the National University of Singapore Institutional Review Board (NUS-IRB).

Informed Consent Informed consent was obtained from all participants prior to their participation.

\section{References}

Adler, J. M., \& Hershfield, H. E. (2012). Mixed emotional experience is associated with and precedes improvements in psychological wellbeing. PLoS One, 7, e35633. https://doi.org/10.1371/journal.pone. 0035633.

Badour, C. L., Resnick, H. S., \& Kilpatrick, D. G. (2017). Associations between specific negative emotions and DSM-5 PTSD among a national sample of interpersonal trauma survivors. Journal of Interpersonal Violence, 32, 1620-1641. https://doi.org/10.1177/ 0886260515589930

Baikie, K. A., Geerligs, L., \& Wilhelm, K. (2012). Expressive writing and positive writing for participants with mood disorders: An online randomized controlled trial. Journal of Affective Disorders, 136, 310-319. https://doi.org/10.1016/j.jad.2011.11.032.

Barford, K. A., Koval, P., Kuppens, P., \& Smillie, L. D. (2020). When good feelings turn mixed: Affective dynamics and big five trait predictors of mixed emotions in daily life. European Journal of Personality, 34, 393-411. https://doi.org/10.1002/per.2264. 
Barford, K. A., \& Smillie, L. D. (2016). Openness and other Big Five traits in relation to dispositional mixed emotions. Personality and Individual Differences, 102, 118-122. https://doi.org/10.1016/j. paid.2016.07.002.

Bauer, J. J., \& McAdams, D. P. (2010). Eudaimonic growth: Narrative growth goals predict increases in ego development and subjective well-being 3 years later. Developmental Psychology, 46, 761-772. https://doi.org/10.1037/a0019654.

Beedie, C., Terry, P., \& Lane, A. (2005). Distinctions between emotion and mood. Cognition \& Emotion, 19, 847-878. https://doi.org/10. 1080/02699930541000057.

Berrios, R., Totterdell, P., \& Kellett, S. (2015). Eliciting mixed emotions: A meta-analysis comparing models, types, and measures. Frontiers in Psychology, 6, e00428. https://doi.org/10.3389/fpsyg.2015. 00428.

Berrios, R., Totterdell, P., \& Kellett, S. (2018). When feeling mixed can be meaningful: The relation between mixed emotions and eudaimonic well-being. Journal of Happiness Studies, 19, 841861. https://doi.org/10.1007/s10902-017-9849-y.

Cohen, J. (1988). Statistical power analysis for the behavioral sciences (2nd ed.). L. Erlbaum Associates.

Curran, P. G. (2016). Methods for the detection of carelessly invalid responses in survey data. Journal of Experimental Social Psychology, 66, 4-19. https://doi.org/10.1016/j.jesp.2015.07.006.

Dalton, C., Corbett, S., \& Katelaris, A. (2020). Pre-emptive low cost social distancing and enhanced hygiene implemented before local COVID-19 transmission could decrease the number and severity of cases. SSRN Electronic Journal. https://doi.org/10.2139/ssrn. 3549276.

Dejonckheere, E., Mestdagh, M., Houben, M., Rutten, I., Sels, L., Kuppens, P., \& Tuerlinckx, F. (2019). Complex affect dynamics add limited information to the prediction of psychological well-being. Nature Human Behaviour, 3, 478-491. https://doi.org/10.1038/ s41562-019-0555-0.

Durso, G. R. O., Briñol, P., \& Petty, R. E. (2016). From power to inaction: Ambivalence gives pause to the powerful. Psychological Science, 27, 1660-1666. https://doi.org/10.1177/ 0956797616669947.

Enders, C., \& Bandalos, D. (2001). The relative performance of full information maximum likelihood estimation for missing data in structural equation models. Structural Equation Modeling: A Multidisciplinary Journal, 8, 430-457. https://doi.org/10.1207/ S15328007SEM0803_5.

Fong, C. T. (2006). The effects of emotional ambivalence on creativity. Academy of Management Journal, 49, 1016-1030. https://doi.org/ 10.5465/amj.2006.22798182.

Forgas, J. P. (2019). Happy believers and sad skeptics? Affective influences on gullibility. Current Directions in Psychological Science, 28, 306-313. https://doi.org/10.1177/0963721419834543.

Forgas, J. P., \& East, R. (2008). On being happy and gullible: Mood effects on skepticism and the detection of deception. Journal of Experimental Social Psychology, 44, 1362-1367. https://doi.org/ 10.1016/j.jesp.2008.04.010.

Fredrickson, B. L. (2001). The role of positive emotions in positive psychology: The broaden-and-build theory of positive emotions. American Psychologist, 56, 218-226. https://doi.org/10.1037/ 0003-066X.56.3.218

Garrett, L. (2020). COVID-19: The medium is the message. The Lancet, 395, 942-943. https://doi.org/10.1016/S0140-6736(20)30600-0.

Gloria, C. T., \& Steinhardt, M. A. (2016). Relationships among positive emotions, coping, resilience and mental health: Positive emotions, resilience and health. Stress and Health, 32, 145-156. https://doi. org/10.1002/smi.2589.

Hart, C. M., Ritchie, T. D., Hepper, E. G., \& Gebauer, J. E. (2015). The balanced inventory of desirable responding short form (BIDR-16).
SAGE Open, 5, 215824401562111. https://doi.org/10.1177/ 2158244015621113.

Hui, C. M., Fok, H. K., \& Bond, M. H. (2009). Who feels more ambivalence? Linking dialectical thinking to mixed emotions. Personality and Individual Differences, 46, 493-498. https://doi.org/10.1016/j. paid.2008.11.022.

Huta, V. (2013). Pursuing eudaimonia versus hedonia: Distinctions, similarities, and relationships. In A. S. Waterman (Ed.), The best within us: Positive psychology perspectives on eudaimonia (pp. 139-158). American Psychological Association. https://doi.org/10.1037/ 14092-008.

Huta, V., \& Waterman, A. S. (2014). Eudaimonia and its distinction from hedonia: Developing a classification and terminology for understanding conceptual and operational definitions. Journal of Happiness Studies, 15, 1425-1456. https://doi.org/10.1007/ s10902-013-9485-0.

Keltner, D., \& Gross, J. J. (1999). Functional accounts of emotions. Cognition \& Emotion, 13, 467-480. https://doi.org/10.1080/ 026999399379140.

Kline, R. B. (2016). Principles and practice of structural equation modeling (4th ed.). Guilford Press.

Lakens, D. (2013). Calculating and reporting effect sizes to facilitate cumulative science: A practical primer for t-tests and ANOVAs. Frontiers in Psychology, 4, e00863. https://doi.org/10.3389/fpsyg. 2013.00863

Landis, R. S., Beal, D. J., \& Tesluk, P. E. (2000). A comparison of approaches to forming composite measures in structural equation models. Organizational Research Methods, 3, 186-207. https:// doi.org/10.1177/109442810032003.

Larsen, J. T., Hemenover, S. H., Norris, C. J., \& Cacioppo, J. T. (2003). Turning adversity to advantage: On the virtues of the coactivation of positive and negative emotions. In L. G. Aspinwall \& U. M. Staudinger (Eds.), A psychology of human strengths: Fundamental questions and future directions for a positive psychology (pp. 211225). https://doi.org/10.1037/10566-015.

Larsen, J. T., Hershfield, H. E., Stastny, B. J., \& Hester, N. (2017). On the relationship between positive and negative affect: Their correlation and their co-occurrence. Emotion, 17, 323-336. https://doi.org/10. 1037/emo0000231.

Larsen, J. T., \& McGraw, A. P. (2011). Further evidence for mixed emotions. Journal of Personality and Social Psychology, 100, 1095-1110. https://doi.org/10.1037/a0021846.

Lerner, J. S., \& Keltner, D. (2001). Fear, anger, and risk. Journal of Personality and Social Psychology, 81, 146-159. https://doi.org/ 10.1037/0022-3514.81.1.146.

Lewnard, J. A., \& Lo, N. C. (2020). Scientific and ethical basis for socialdistancing interventions against COVID-19. The Lancet Infectious Diseases, S1473309920301900, 631-633. https://doi.org/10.1016/ S1473-3099(20)30190-0.

McCarthy, J. (2020). U.S. Emotions Mixed After a Tense Month of COVID-19 Response. Gallup Panel. https://news.gallup.com/poll/ 306026/emotions-mixed-tense-month-covid-response.aspx

Mejía, S. T., \& Hooker, K. (2017). Mixed emotions within the context of goal pursuit. Current Opinion in Behavioral Sciences, 15, 46-50. https://doi.org/10.1016/j.cobeha.2017.05.015.

Miyamoto, Y., \& Ryff, C. D. (2011). Cultural differences in the dialectical and non-dialectical emotional styles and their implications for health. Cognition \& Emotion, 25, 22-39. https://doi.org/10.1080/ 02699931003612114

Newman, D. B., Sachs, M. E., Stone, A. A., \& Schwarz, N. (2019). Nostalgia and well-being in daily life: An ecological validity perspective. Journal of Personality and Social Psychology, 118, 325347. https://doi.org/10.1037/pspp0000236.

Pasion, R., Paiva, T. O., Fernandes, C., \& Barbosa, F. (2020). The age effect on protective behaviors during the covid-19 outbreak: Sociodemographic, perceptions and psychological accounts. 
Frontiers in Psychology, 11. https://doi.org/10.3389/fpsyg.2020. 561785.

Permut, S., Fisher, M., \& Oppenheimer, D. M. (2019). Taskmaster: A tool for determining when subjects are on task. Advances in Methods and Practices in Psychological Science, 2, 188-196. https://doi.org/ $10.1177 / 2515245919838479$.

Rees, L., Rothman, N. B., Lehavy, R., \& Sanchez-Burks, J. (2013). The ambivalent mind can be a wise mind: Emotional ambivalence increases judgment accuracy. Journal of Experimental Social Psychology, 49, 360-367. https://doi.org/10.1016/j.jesp.2012.12. 017.

Robinson, M. D., \& Clore, G. L. (2002). Episodic and semantic knowledge in emotional self-report: Evidence for two judgment processes. Journal of Personality and Social Psychology, 83, 198-215. https:// doi.org/10.1037/0022-3514.83.1.198.

Ryan, R. M., \& Deci, E. L. (2001). On happiness and human potentials: A review of research on hedonic and eudaimonic well-being. Annual Review of Psychology, 52, 141-166. https://doi.org/10.1146/ annurev.psych.52.1.141.

Selby, E. A., Kranzler, A., Panza, E., \& Fehling, K. B. (2016). Bidirectional-compounding effects of rumination and negative emotion in predicting impulsive behavior: Implications for emotional cascades: bidirectional-compounding rumination. Journal of Personality, 84, 139-153. https://doi.org/10.1111/jopy.12147.

Timmons, S., McGinnity, F., Belton, C., Barjaková, M., \& Lunn, P. (2020). It depends on how you ask: Measuring bias in population surveys of compliance with COVID-19 public health guidance. Journal of Epidemiology and Community Health, 75, 387-389. https://doi.org/10.1136/jech-2020-215256.
Tran, H. N., Lipinski, A. J., Peter, S. C., Dodson, T. S., Majeed, R., Savage, U. C., \& Beck, J. G. (2019). The association between posttraumatic negative self-conscious cognitions and emotions and maladaptive behaviors: Does time since trauma exposure matter? Journal of Traumatic Stress, 32, 249-259. https://doi.org/10.1002/ jts. 22388 .

Vaccaro, A. G., Kaplan, J. T., \& Damasio, A. (2020). Bittersweet: The neuroscience of ambivalent affect. Perspectives on Psychological Science, 174569162092770, 1187-1199. https://doi.org/10.1177/ 1745691620927708

van Harreveld, F., Nohlen, H. U., \& Schneider, I. K. (2015). The ABC of ambivalence: affective, behavioral, and cognitive consequences of attitudinal conflict. In Advances in Experimental Social Psychology (Vol. 52, pp. 285-324). Elsevier. https://doi.org/10.1016/bs.aesp. 2015.01.002.

van Harreveld, F., van der Pligt, J., \& de Liver, Y. N. (2009). The agony of ambivalence and ways to resolve it: Introducing the MAID model. Personality and Social Psychology Review, 13, 45-61. https:// doi.org/10.1177/1088868308324518.

Young, E. S., Griskevicius, V., Simpson, J. A., Waters, T. E. A., \& Mittal, C. (2018). Can an unpredictable childhood environment enhance working memory? Testing the sensitized-specialization hypothesis. Journal of Personality and Social Psychology, 114, 891-908. https://doi.org/10.1037/pspi0000124.

Ziegler, R. (2014). Mood and processing effort. In Advances in Experimental Social Psychology (Vol. 49, pp. 287-355). Elsevier. https://doi.org/10.1016/B978-0-12-800052-6.00005-6. 